\title{
Predicted Effects of a Pause in D1 and D2 Medium Spiny Neurons during Feeding
}

\author{
Cristianne R. M. Frazier ${ }^{1}$ and Ana Mrejeru ${ }^{2}$ \\ ${ }^{1}$ Committee on Neurobiology, University of Chicago, Chicago, Illinois 60637 and ${ }^{2}$ Department of Neurology, Columbia University Medical Center, New \\ York, New York 10032 \\ Review of Krause et al.
}

A reduction in nucleus accumbens (NAc) activity is associated with an increase in feeding under several experimental conditions. In the late 1990s, Kelley et al. reported that sated rats ate "voraciously" when an AMPA antagonist or $\mathrm{GABA}_{\mathrm{A}}$ agonist was infused into the NAc shell, an effect specific to feeding, not water consumption or chewing movements (reviewed by Kelly et al., 2005). Later, Roitman et al. found that infusing sucrose directly into rats' mouths was correlated with a decrease in the firing rate of NAc neurons (Roitman et al., 2005). However, neither line of work addressed whether a reduction in NAc neuron firing is necessary for feeding to occur. A recent study by Krause et al. (2010) published in The Journal of Neuroscience tested this by electrically stimulating neurons while rats licked a spout for sugar water. The authors found that stimulating certain sites in the NAc shell and NAc core transiently stopped licking behavior, and concluded

Received May 28, 2010; revised June 16, 2010; accepted June 17, 2010.

Preparation of this manuscript was supported by award number F31DA026802 from National Institute on Drug Abuse (NIDA) (to C.R.M.F.) and Training Grant 5T32DA007255-18 from NIDA (to A.M.). The Gene Expression Nervous System Atlas Project was conducted under National Institute of Neurological Disorders and Stroke Contracts N01NS02331 and HHSN271200723701C to The Rockefeller University (New York, NY). We are grateful to Xiaoxi Zhuang for his feedback on this manuscript, and to Clifton Ragsdale and Jeff Beeler for enlightening discussions.

Correspondence should be addressed to Cristianne R. M. Frazier, $924 \mathrm{E}$. 57th Street, Room 222, Chicago, IL 60637. E-mail: frazierc@uchicago.edu. DOI:10.1523/JNEUROSCI.2745-10.2010

Copyright $\odot 2010$ the authors $\quad 0270-6474 / 10 / 309964-03 \$ 15.00 / 0$ that a pause in NAc neuron firing is necessary for feeding behavior to occur.

There are several types of neurons in the NAc, and so the following question arises: which neurons must pause to maintain feeding behavior? Although the authors did not speculate about the biochemical nature of the neurons they stimulated, it is likely that stimulation affected GABAergic medium spiny neurons (MSNs), the most abundant cell type in the NAc (>90\%) and the only neurons that project out of this region. There are two main types of MSNs: those containing D1-like receptors and those that express D2-like dopamine receptors; colocalization of D1-like and D2-like receptors occurs in $<1 \%$ of NAc MSNs (Shuen et al., 2008). Is a pause in both D1 and D2 MSNs required for feeding behavior, or is a pause in one type more critical than the other? We will discuss the known projections of D1 and D2 MSNs and how a pause in each type is predicted to effect feeding.

The projections of MSNs from the NAc core are similar to those from the dorsal striatum (Nicola, 2007; Humphries and Prescott, 2010). D1 MSNs in both areas excite the motor thalamus via a direct pathway, whereas D2 MSNs inhibit the motor thalamus via an indirect pathway. The main difference between the NAc core and dorsal striatum projections is that D2 MSNs of the NAc core project to the dorsolateral portion of the ventral pallidum (VP) (Fig. 1E), whereas those of the dorsal striatum project to the globus pallidus.

Activation of D2 MSNs in either the NAc core or the dorsal striatum ultimately reduces excitatory drive to motor thalamus, and thus inhibits movement. Therefore, a pause in some D2 MSNs in NAc core is expected to promote licking movements. Conversely, stimulating some of these D2 MSNs during an ongoing licking behavior is predicted to inhibit movement. We suspect that stimulation of D2 MSNs likely mediates the reduction in feeding seen by Krause et al. (2010).

In contrast to D2 MSNs, activation of D1 MSNs of the NAc core stimulates motor thalamus and promotes movement (Fig. $1 E$ ). A pause in D1 MSNs would reduce licking movements, whereas stimulating some of these neurons would likely enhance motor output. Therefore, stimulation of D1 MSNs is unlikely to mediate the reduction in feeding seen by Krause et al. (2010).

Understanding how a pause in NAc shell neurons maintains feeding is more complicated than for NAc core, because the projections of D1 and D2 MSNs from NAc shell are not well characterized. However, it is established that glutamate neurons in the lateral hypothalamus ( $\mathrm{LH})$ are a major target of MSNs, and the NAc shell is the only striatal region that sends projections to LH (Mogenson et al., 1983; Heimer et al., 1991; Sano and Yokoi, 2007). The LH is an orexigenic region that projects to brainstem nuclei important for 


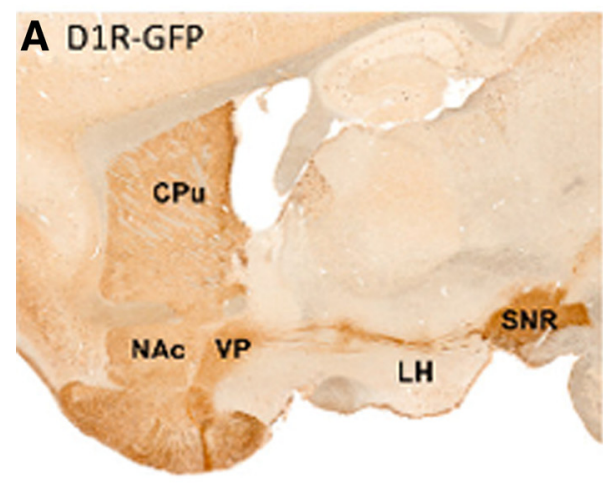

C D2R-GFP

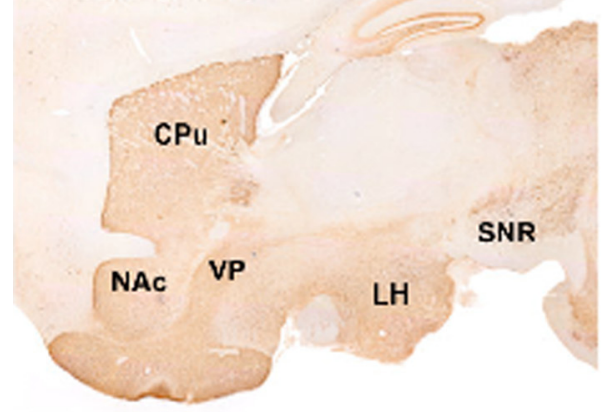

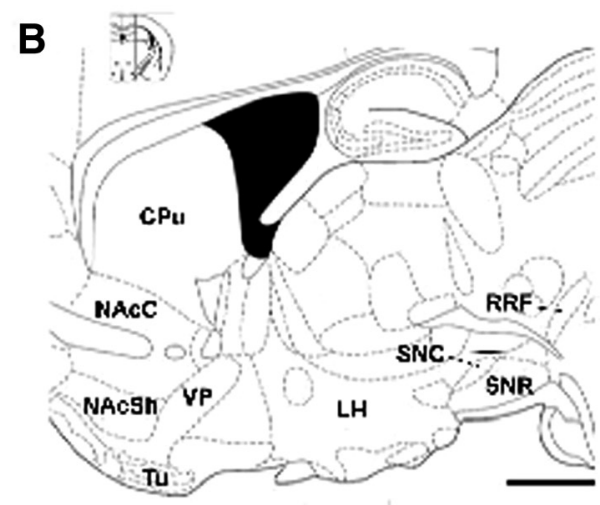

D D2R MRNA

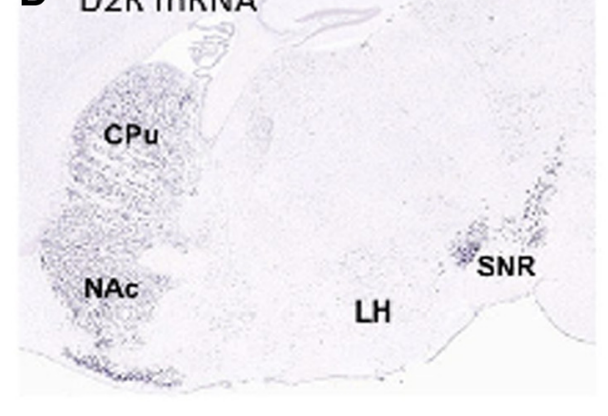

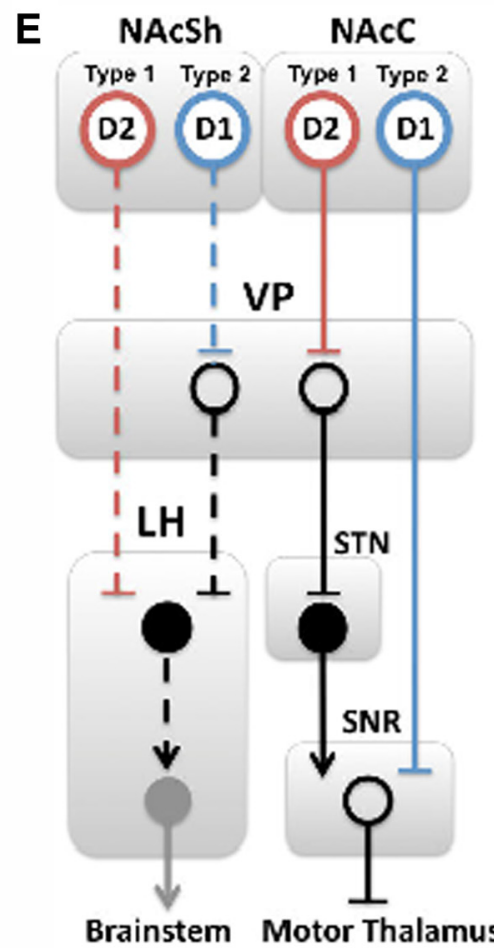

Figure 1. $\boldsymbol{A}, \boldsymbol{C}$, Projections of D1-containing $(\boldsymbol{A})$ and D2-containing $(\boldsymbol{C})$ neurons in mouse brain, courtesy of GENSAT. $\boldsymbol{B}$, Anatomical locations at interaural $1.08 \mathrm{~mm}$ defined by Paxinos and Franklin (2001). Bar = $1 \mathrm{~mm}$. D, In situ hybridization for D2 mRNA, courtesy of the Allen Mouse Brain Atlas (Allen Institute for Brain Research, 2009). $\boldsymbol{E}$, Simplified schematic of the known (solid lines) and hypothesized (dashed lines) output pathways of the nucleus accumbens. We propose that the type 1 neurons defined by Krause et al. (2010) are D2 MSNs and the type 2 neurons are D1 MSNs. 0pen circles, inhibitory neurons; filled circles, excitatory neurons. CPu, Caudate-putamen; NAc (Sh/C), nucleus accumbens (shell/core); Tu, olfactory tubercle; SN (R/C), substantia nigra (reticulata/ compacta); RRF, retrorubral field; STN, subthalamic nucleus.

feeding-related motor patterns and autonomic arousal (Kelley et al., 2005). MSNs from the NAc shell also project to the medial ventral pallidum, which inhibits neurons in the LH (Heimer et al., 1991; Groenewegen et al., 1993). Thus, it appears that "direct" and "indirect" pathways from the NAc shell to the LH may exist. Whether these pathways originate with D1 and D2 MSNs, as they do in the NAc core and dorsal striatum, has not been explored (Humphries and Prescott, 2010).

We examined the Gene Expression Nervous System Atlas (GENSAT) image database of D1 and D2 neurons expressing green fluorescent protein from BAC transgenic mice and found that D2 neurons appear to send robust projections directly to $\mathrm{LH}$ (Fig. $1 C, D$, plus adjacent sections not shown here). However, D1 neurons do not appear to project robustly to LH (Fig. $1 A$, plus adjacent sections not shown here). This suggests that a direct pathway from the NAc shell to the LH may originate with D2 MSNs, and an indirect pathway, potentially via the medial ventral pallidum, might originate with D1 MSNs (Fig. $1 E$ ). This is consistent with a report showing that D1 MSNs from the NAc shell project to the VP (Lu et al.,
1998). This anatomy may be the reverse of NAc core and dorsal striatum output pathways, but the functional effects are likely to be similar. A pause in some D2 MSNs in the NAc shell is predicted to promote activity in the LH and maintain feeding, whereas a pause in some D1 MSNs is predicted to reduce feeding. This is a relatively simple model of the NAc to LH projection; detailed anatomical and functional studies are needed to determine the output pathways of D1 and D2 MSNs from the NAc shell and their functions.

The role of the NAc shell and NAc core in feeding and other appetitive behaviors is still hotly debated (Humphries and Prescott, 2010), but Kelley et al. (2005) have suggested that the NAc shell is critical for basic consumption and foraging behaviors, whereas the NAc core is more important for relatively complex appetitive tasks, which may involve pavlovian learning. The feeding task used by Krause et al. (2010) required that the animal learn to associate a cue with the availability of sucrose (pavlovian learning) and then perform an operant task to acquire the sucrose (moving to the opposite side of the cage to lick a spout). It is possible that both the NAc shell and NAc core have a role to play in the feeding behavior elicited by this task, and this may explain why effective stimulation sites were found in both regions.

Krause et al. (2010) elegantly described two populations of neurons based on firing properties before, during, and after rats licked a spout for sugar water. These two types have firing patterns that bear a striking resemblance to the expected firing patterns of D2 and D1 MSNs during this task. Type 1 neurons increased their firing rate from $\sim 4$ to $15 \mathrm{~Hz}$ after a cue signaling sucrose availability was presented. Then, they began to pause $\sim 1 \mathrm{~s}$ before the rat initiated its first lick on the sugar spout (Krause et al., 2010, their Figs. 2,3 ) and remained quiescent while the animal licked the spout even if sucrose was no longer being dispensed (Krause et al., 2010, their Fig. 4). Based on these findings, the authors concluded that type 1 neurons encode the motor program required for consumption of sucrose. This conclusion contrasts with previous reports interpreting a pause in NAc neurons as encoding the rewarding aspects of a substance (Roitman et al., 2005) (see discussion by Krause et al., 2010). Furthermore, Krause et al. (2010) found that the stimulation sites in the NAc that were 
most effective at inhibiting licking for sucrose overlapped significantly with the sites where type 1 neurons were recorded (Krause et al., 2010, their Fig. 8). This suggests that a pause in type 1 neurons in the NAc is required to maintain feeding. Even though the authors found type 1 cells and effective stimulation cites in the dorsal striatum, these sites do not appear to overlap. Therefore, it is unclear whether a pause in dorsal striatum MSNs is required to maintain feeding in the task used by Krause et al. (2010).

Based on the anatomical evidence described above suggesting that a pause in D2 MSNs, but not D1 MSNs, maintains feeding behavior, we propose that type 1 neurons are D2 MSNs (Fig. $1 E$ ). It is interesting that type 1 neurons increased their firing rate during cue presentation and before pausing. If these are D2 MSNs, this burst of activity may inhibit a current action so that another action, in this case licking behavior, can commence. These neurons may then pause to prevent further inhibition and maintain feeding. We predict, however, that without the ramping phase and inhibition of the current action, feeding behavior would not be initiated. The function of D2 MSN firing in the NAc shell and NAc core during the preparatory versus consumption phases of feeding could be tested using optogenetic approaches (Gradinaru et al., 2008).

Krause et al. (2010) described a second type of neuron that remained active at 3-6 Hz during the cue presentation, and then increased firing rate when type 1 neurons began to pause, and/or during licking behavior (Krause et al., 2010, their Fig. 2) (supplemental Fig. 1, available at www.jneurosci.org as supplemental ma- terial). We propose that type 2 neurons are D1 MSNs, because an increase in D1 MSN firing rate is predicted to promote motor behavior: licking in this case. In fact, Krause et al. (2010) found that low levels of stimulation actually increased lick frequency (Krause et al., 2010, their Fig. 5). Because D1 MSNs are slightly more abundant than D2 MSNs (35 vs $26 \%$, respectively) (Shuen et al., 2008), stimulation may initially recruit some D1 MSNs that promote feeding behavior. However, more robust stimulation could activate enough type 1 neurons/putative D2 MSNs to put the brake on the motor systems needed for feeding. Even if D1 MSNs fire during high levels of stimulation, the effect may be akin to revving an engine: the system is not likely to produce feeding if the brakes are on.

What is the role of dopamine in this system? Dopamine release during the training/learning phase of this feeding task may be important for setting up the synaptic weights that produce a pause in the appropriate D2 MSNs and increased firing rates in the appropriate D1 MSNs when a cue predicting sucrose availability is presented and consumption is initiated. The molecular identification of type 1 and 2 neurons would merge this impressive in vivo work with studies on the role of dopamine signaling in MSN plasticity and reward learning.

\section{References}

Allen Institute for Brain Science (2009) Allen mouse brain atlas. Seattle, WA: Allen Institute for Brain Science. Available at http:// mouse.brain-map.org.

Gradinaru V, Thompson KR, Deisseroth K (2008) eNpHR: a Natronomonas halorhodopsin enhanced for optogenetic applications. Brain Cell Biol 36:129-139.

Groenewegen HJ, Berendse HW, Haber SN
(1993) Organization of the output of the ventral striatopallidal system in the rat: ventral pallidal efferents. Neuroscience 57:113-142.

Heimer L, Zahm DS, Churchill L, Kalivas PW, Wohltmann C (1991) Specificity in the projection patterns of accumbal core and shell in the rat. Neuroscience 41:89-125.

Humphries MD, Prescott TJ (2010) The ventral basal ganglia, a selection mechanism at the crossroads of space, strategy, and reward. Prog Neurobiol 90:385-417.

Kelley AE, Baldo BA, Pratt WE, Will MJ (2005) Corticostriatal-hypothalamic circuitry and food motivation: integration of energy, action and reward. Physiol Behav 86:773-795.

Krause M, German PW, Taha SA, Fields HL (2010) A pause in nucleus accumbens neuron firing is required to initiate and maintain feeding. J Neurosci 30:4746-4756.

Lu XY, Ghasemzadeh MB, Kalivas PW (1998) Expression of D1 receptor, D2 receptor, Substance $\mathrm{P}$ and enkephalin messenger RNAs in the neurons projecting from the nucleus accumpens. Neuroscience 82:767-780.

Mogenson GJ, Swanson LW, Wu M (1983) Neural projections from nucleus accumbens to globus pallidus, substantia innominata, and lateral preopticlateral hypothalamic area: an anatomical and electrophysiological investigation in the rat. J Neurosci 3:189-202.

Nicola SM (2007) The nucleus accumbens as part of a basal ganglia action selection circuit. Psychopharmacology (Berl) 191:521-550.

Roitman MF, Wheeler RA, Carelli RM (2005) Nucleus accumbens neurons are innately tuned for rewarding and aversive taste stimuli, encode their predictors, and are linked to motor output. Neuron 45:587-597.

Sano H, Yokoi M (2007) Striatal medium spiny neurons terminate in a distinct region in the lateral hypothalamic area and do not directly innervate orexin/hypocretin- or melaninconcentrating hormone-containing neurons. J Neurosci 27:6948-6955.

Shuen JA, Chen M, Gloss B, Calakos N (2008) Drdla-tdTomato BAC transgenic mice for simultaneous visualization of medium spiny neurons in the direct and indirect pathways of the basal ganglia. J Neurosci 28:2681-2685. 